Review

\title{
Deep-Sea Fungi Could Be the New Arsenal for Bioactive Molecules
}

\author{
Muhammad Zain ul Arifeen, Yu-Nan Ma, Ya-Rong Xue and Chang-Hong Liu * \\ State Key Laboratory of Pharmaceutical Biotechnology, School of Life Sciences, Nanjing University, \\ Nanjing 210023, China; m.z.arifeen@gmail.com (M.Z.u.A.); mayunan994727@163.com (Y.-N.M.); \\ xueyr@nju.edu.cn (Y.-R.X.) \\ * Correspondence: chliu@nju.edu.cn; Tel./Fax: +86-25-8968-5469
}

Received: 28 November 2019; Accepted: 17 December 2019; Published: 20 December 2019

check for updates

\begin{abstract}
Growing microbial resistance to existing drugs and the search for new natural products of pharmaceutical importance have forced researchers to investigate unexplored environments, such as extreme ecosystems. The deep-sea ( $>1000 \mathrm{~m}$ below water surface) has a variety of extreme environments, such as deep-sea sediments, hydrothermal vents, and deep-sea cold region, which are considered to be new arsenals of natural products. Organisms living in the extreme environments of the deep-sea encounter harsh conditions, such as high salinity, extreme $\mathrm{pH}$, absence of sun light, low temperature and oxygen, high hydrostatic pressure, and low availability of growth nutrients. The production of secondary metabolites is one of the strategies these organisms use to survive in such harsh conditions. Fungi growing in such extreme environments produce unique secondary metabolites for defense and communication, some of which also have clinical significance. Despite being the producer of many important bioactive molecules, deep-sea fungi have not been explored thoroughly. Here, we made a brief review of the structure, biological activity, and distribution of secondary metabolites produced by deep-sea fungi in the last five years.
\end{abstract}

Keywords: deep-sea; extreme; ecosystem; fungi; bioactive compounds; secondary metabolites

\section{Deep-Sea Fungi: A Novel Source of Bioactive Molecules}

Antibiotics and antifungal drugs are the most commonly used drugs in the world, but their role in treating human diseases has been greatly reduced due to the development of pathogen resistance against these drugs. Scientists are now looking for new, untapped and renewable resources for the isolation of novel compounds to with clinical importance. Despite the fact that the ocean provides habitats to a huge number of microbes, both fungi and bacteria for thousands of years, the microbes of these extreme ecosystems and their potential for new drug discovery have not yet been fully realized due to methodological and technical limitations. Fungi are the most diverse and abundant eukaryotic organisms on the planet, and their presence in all possible extreme ecosystems make them an ideal source for investigations of new drug development. Scientists are interested in the extraction of novel and unique natural products, having clinical importance, from different organisms living in the extreme environments. In addition to terrestrial extreme environments, the ocean could also be considered a good reservoir of bioactive metabolites [1-4]. Fungi living in the deep-sea environments are known to produce novel bioactive compounds. Although, it is not fully understood why the fungi living in the extreme environments produce unique and novel products, it is assumed that fungal genome has evolved to make necessary adjustments in order to sustain life in such harsh conditions and might be involved in chemical defense and communication [5].

The ocean is considered to be one of the most diverse ecosystems. Compared to terrestrial and coastal ecosystems, the deep-sea (water depths below $1000 \mathrm{~m}$ ) has a variety of extreme environments, 
such as temperatures ranging from 0 to $400{ }^{\circ} \mathrm{C}$, lack of light and oxygen, high hydrostatic pressure up to $400 \mathrm{~atm}$, and limited supply of nutrient substrates, making these habitats extremely difficult for life [6,7]. In order to inhabit such extreme ecosystems, organisms should have the potential to adjust to these conditions with different mechanism, such as regulating temperature, $\mathrm{pH}$, and solute concentration, as well as the production of biomolecules to control DNA, protein, and lipid damage. This may be why microorganisms growing in these environments produce special metabolites.

Previously, drug investigators mainly considered bacteria, especially actinomycetes, as an important source of antifungal and antibacterial drugs. Cephalosporin $C$ was the first compound derived from the marine fungus Cephalosporium sp. in 1949. After that, a number of important drugs - for instance, polyketide griseofulvin, terpenoid fusidic acid, cephalosporins, etc.- have been isolated from the marine fungi. Despite being the source of such important products, deep-sea fungi have not received full attention [8]. With the increasing demand for new drugs, scientists are now looking for new and unexplored resources for bioactive compounds, and the deep-sea consists of some extreme ecosystems that are worth exploring for new metabolites. Studies about isolating new bioactive molecules from marine environments are growing at an increasing rate, and hundreds of new compounds are reported every year; for instance, in 2017, a total of 448 new compounds were reported [9].

In this review, we present an overview of all those new and important bioactive metabolites isolated from deep-sea fungi during the last five years. We include only those molecules which were extracted from the deep-sea fungi associated with some kind of extreme environments, irrespective of its isolation from terrestrial counterparts, while all those compounds were excluded which were isolated from marine fungi and were not associated with extreme environments. This review will benefit all those who are interested in extreme-marine-environment fungi and their bioactive molecules. For more detailed information about other important secondary metabolites extracted from marine fungi, one should refer to our previous review papers [10-12].

\section{Bioactive Compounds from Deep-Sea Fungi}

According to the literature survey, we found 151 novel bioactive compounds isolated from marine fungi extracted from different extreme environments in the last five years. The majority of these compounds were isolated from two fungal genera i.e., Penicillium $(63,41.2 \%$ of the total compounds) and Aspergillus (43, 28.1\% of the total compounds). Table 1 lists the detail of these compounds, which fall into different categories according to their structure.

\subsection{Polyketide Compounds}

Twenty-four polyketide compounds (1-24; Figure 1) with important biological activities were isolated from fungi extracted from different deep-sea environments. Among them, compounds 1 and 2 were isolated from Penicillium spp., which showed antibiotic activity (MIC of $32 \mu \mathrm{g} / \mathrm{mL}$ against Bacillus subtilis) and nuclear factor NF-kB inhibition activity, respectively $[13,14]$. Compounds 3-11 were from Aspergillus sp. 16-02-1, which exhibited cytotoxicity (with a 10\%-80\% inhibition rate at $100 \mu \mathrm{g} / \mathrm{mL}$ against various cancer cell lines i.e., K562, HL-60, HeLa, and BGC-823) [15]. Similarly, compounds 12-24 were isolated from the species belonging to Ascomycetes, Engyodontium, and Lindgomycetaceae, out of which compounds 12-13 and 23-24 showed strong antibiotic activities against Bacillus subtilis, Acinetobacter baumannii, Escherichia coli, Staphylococcus aureus, Enterococcus faecalis, Staphylococcus epidermidis, and Propionibacterium acnes, while compounds 14-22 exhibited strong cytotoxic activity $\left(\mathrm{IC}_{50} 4.9 \mu \mathrm{M}\right)$ against U937 cells (Table 1) [16-18]. 
<smiles>COc1c(C)c([C@](C)(O)C(OC)/C(C)=C/C(C)=C/[C@@]2(C)O[C@H](C)C3(C)O[C@@H]32)oc(=O)c1C</smiles>

Methyl isoverrucosidinol (1)

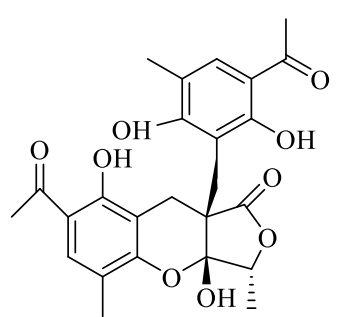

Penilactone A (2)<smiles>C=C(C)CC1=C[C@@H]([C@H](C)O)OC1=O</smiles>

Aspiketolactonol (3)<smiles>C[C@H](O)CCC1=C[C@@H](C)OC1=O</smiles>

Aspilactonol A (4)<smiles>CO[C@H](C1=C[C@@H](C(C)O)OC1=O)[C@@H](C)O</smiles>

Aspilactonol D (7)<smiles>[H][Z]1(C)cc(C(O)C(C)O)c(=O)o1</smiles>

Aspilactonol B 8S, 9R (5) Aspilactonol C 8R, 9S (6)

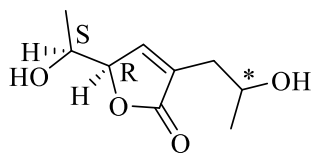

Aspilactonol E 8S, 9R (8) Aspilactonol F 8R, 9S (9)<smiles>CO[C@H](C1=C[C@@H](O)[C@H](C)OC1=O)[C@H](C)O</smiles>

Aspyronol (10)<smiles>CCC(C=CC(C)=O)=C[C@H](C)O</smiles>

Epiaspinonediol (11)<smiles>COC(=O)c1cc(OC)c(O)c2c1C1=C(CO2)C(=O)C[C@H](C)O1</smiles>

Ascomycotin A (12)<smiles>[R]OC(=O)c1c([R])ccc2oc3cc([R3])cc(O)c3c(=O)c12</smiles>

Engyodontiumone $\mathrm{A}, \mathrm{R}_{1}=\mathrm{CH}_{3}$, $\mathrm{R}_{2}=\mathrm{OCH}_{3}, \mathrm{R}_{3}=\mathrm{CH}_{2} \mathrm{OH}$ (14) Engyodontiumone $\mathrm{B}, \mathrm{R}_{1}=\mathrm{CH}_{3}$, $\mathrm{R}_{2}=\mathrm{Cl}, \mathrm{R}_{3}=\mathrm{CH}_{2} \mathrm{OH}$ (15)<smiles>COC(=O)[C@H]1c2c(oc3cc(CO)cc(O)c3c2=O)CC(O)C1O</smiles>

Engyodontiumone F (19)<smiles>C/C(=C\CCC(C)(C)O)c1ccc(C(=O)O)cc1O</smiles>

Engyodontiumone J (22)<smiles>COC(=O)[C@H]1c2c(oc3cc(CO)cc(O)c3c2=O)CC[C@H]1O</smiles>

Engyodontiumone C (16)

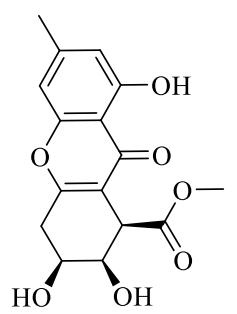

Engyodontiumone D (17)<smiles>Cc1cc(O)cc(Oc2cc(C)cc(O)c2)c1</smiles>

Diorcinol (13)

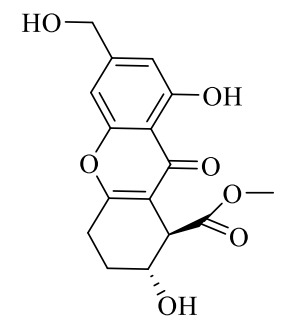

Engyodontiumone E (18)<smiles>COC(=O)C1c2c(oc3cc(CO)cc(O)c3c2=O)CC(O)C1O</smiles>

Engyodontiumone G (20)

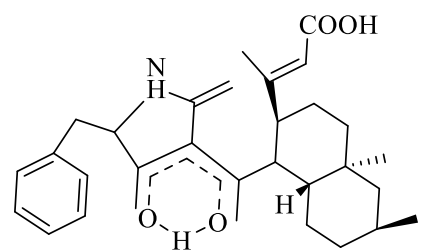

Lindgomycin (23)<smiles>CC(=CCCC(C)(C)O)c1ccc(C(=O)O)cc1O</smiles>

Engyodontiumone I (21)

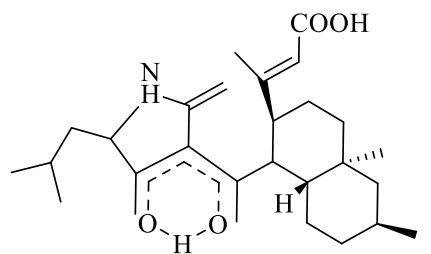

Ascosetin (24)

Figure 1. Structures of polyketide secondary metabolites obtained from deep-sea fungi.

\subsection{Nitrogen-Containing Compounds}

Twenty-four novel alkaloid-bioactive compounds (25-48; Figure 2) have been reported from deep-sea fungi since 2013, out of which compounds 25-40 were isolated from Penicillium spp., and showed cytotoxic activities against BV2 cell ( $\mathrm{IC}_{50}$ of $\left.27-45 \mu \mathrm{g} / \mathrm{mL}\right)$, brine shrimp (IC 50 of 14.1 to $38.5 \mu \mathrm{g} / \mathrm{mL}$ ), SMMC-7721 (IC 50 of $54.2 \mu \mathrm{M})$, BEL-7402 ((IC 50 of $17.5 \mu \mathrm{M})$, and BEL-7402 (IC 50 of $19.8 \mu \mathrm{M}$ ) [19-21]. Compounds 41-46 were identified from Aspergillus spp., in which compounds 41 and 45-46 displayed antibiotic activity (MIC of 30 to $40 \mu \mathrm{g} / \mathrm{mL}$ ) against BCG, Candida albicans, Bacillus subtilis, Staphylococcus aureus, Pseudomonas aeruginosa, Bacillus cereus, Klebsiella pneumoniae, and Escherichia coli, 
while compounds 47 and 48 were extracted from other genera and showed antimicrobial activity (MIC between 16 and $64 \mu \mathrm{g} / \mathrm{mL}$ against Escherichia coli, Aeromonas hydrophila, Micrococcus luteus, Staphylococcus aureus, Vibrio anguillarum, Vibrio harveyi, and Vibrio parahaemolyticus) and cytotoxic activity against human cervical carcinoma HeLa, respectively [22-26].

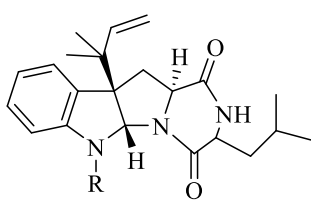

Brevicompanines D, $\mathrm{R}=\mathrm{CH}_{2} \mathrm{OCH}_{3}$ (25) Brevicompanines E, $\mathrm{R}=\mathrm{COCH}_{2} \mathrm{CH}_{3}$ (26) Brevicompanines $\mathrm{F}, \mathrm{R}=\mathrm{CO}\left(\mathrm{CH}_{2}\right)_{2} \mathrm{CH}_{3}$ (27) Brevicompanines $\mathrm{G}, \mathrm{R}=\mathrm{COCH}_{3}$ (28) Brevicompanines $\mathrm{H}, \mathrm{R}=\mathrm{CH}_{2} \mathrm{COCH}_{3}$ (29)

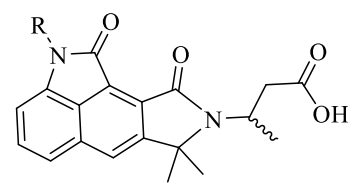

Cyclopiamide $\mathrm{B}, \mathrm{R}=\mathrm{CH}_{3}$ (30) Cyclopiamide $\mathrm{C}, \mathrm{R}=\mathrm{H}$ (31)

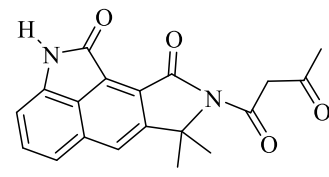

Cyclopiamide D (32)<smiles>Cc1cc(=O)n2c(n1)-c1c(cc3cccc4c3c1C(=O)N4C)C2(C)C</smiles>

Cyclopiamide E (33)

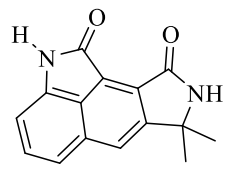

Cyclopiamide F (34)

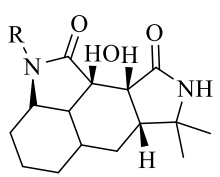

Cyclopiamide $\mathrm{G}, \mathrm{R}=\mathrm{H}(\mathbf{3 5})$ Cyclopiamide $\mathrm{H}, \mathrm{R}=\mathrm{CH}_{3}(\mathbf{3 6})$

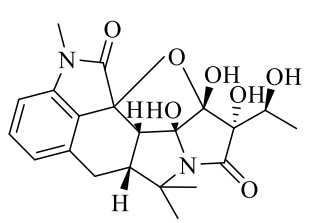

Cyclopiamide I (37)
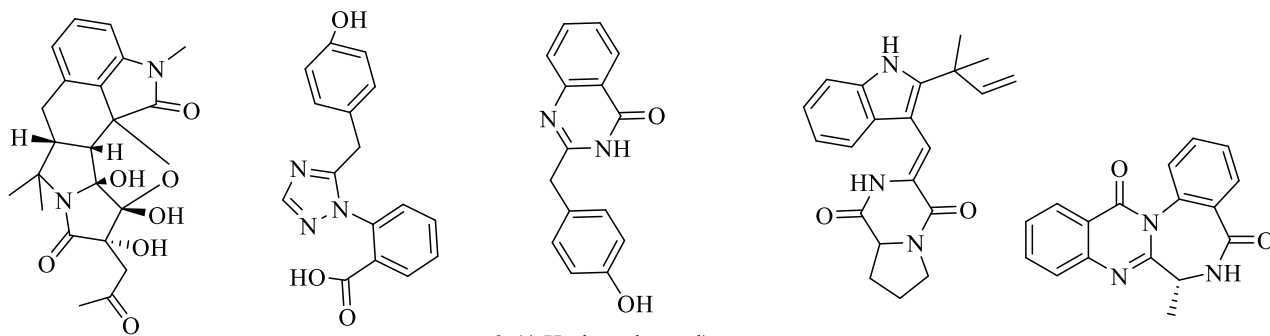

Cyclopiamide J (38)

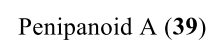

2-(4-Hydroxybenzyl) quinazolin-4(3H)-one $(40)$

( \pm Brevianamide R (41)

Circumdatin F (42)
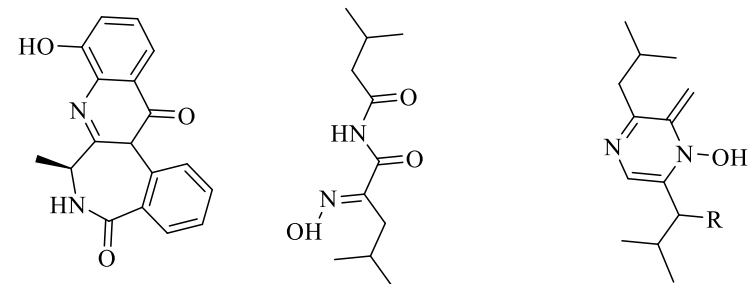

Circumdatin G (43) $\quad$ Oximoaspergillimide (44) $\begin{gathered}\text { Neohydroxyaspergillic, } \mathrm{R}=\mathrm{OH} \text { (45) } \\ \text { Neoaspergillic, } \mathrm{R}=\mathrm{H}(\mathbf{4 6})\end{gathered}$

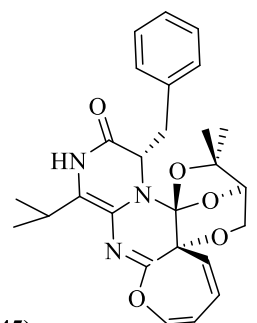

Varioxepine A (47)

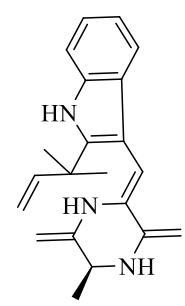

Neoechinulin A (48)

Figure 2. Bioactive alkaloid compounds isolated from deep-sea fungi.

\subsection{Polypeptides}

Twenty-two polypeptides with novel structures (49-70; Figure 3) were reported from fungi inhabiting different marine environments during 2013-2019. Compounds 49 and 50 were isolated from Penicillium canescens and displayed antibiotic activity against Bacillus amyloliquefaciens and Pseudomonas aeruginosa at $100 \mu \mathrm{M}$, while compounds 51-55 were extracted from Aspergillus spp., in which 51-54 showed cytotoxic activity ( $\mathrm{IC}_{50}$ of 15-25 $\mu \mathrm{g} / \mathrm{mL}$ ) against HepG2, SMMC-7721, Bel-7402, and human glioma U87 cell lines, while compound 55 showed inhibitory effects $\left(\mathrm{IC}_{50}\right.$ value of $5.11 \mu \mathrm{mol} / \mathrm{L}$ ) against Mycobacterium tuberculosis protein tyrosine phosphatase B (MptpB) [27-30]. However, compounds 56-64, which were obtained from Simplicillium obclavatum, and 65-70, obtained from Trichoderma asperellum, displayed cytotoxicity ( $\mathrm{IC}_{50}$ of 39.4-100 $\mu \mathrm{M}$ ) against human leukemia HL-60 and K562 cell lines and antibiotic activity ( $\mathrm{IC}_{50}$ of 39.4-100 $\left.\mu \mathrm{M}\right)$ against Gram-positive bacteria 
(e.g., Bacillus amyloliquefaciens, Staphylococcus aureus) and Gram-negative bacteria (e.g., Pseudomonas aeruginosa and Escherichia coli), respectively [28,31].
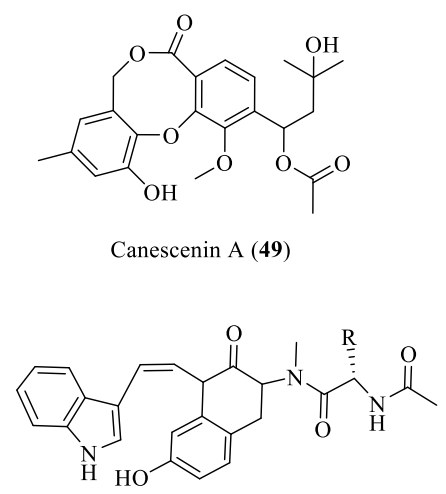<smiles>CCC(C)C(=O)O[C@H]1O[C@](C)(C(=O)OC)C(=O)[C@H](O)C1=O</smiles>

Canescenin B (50)
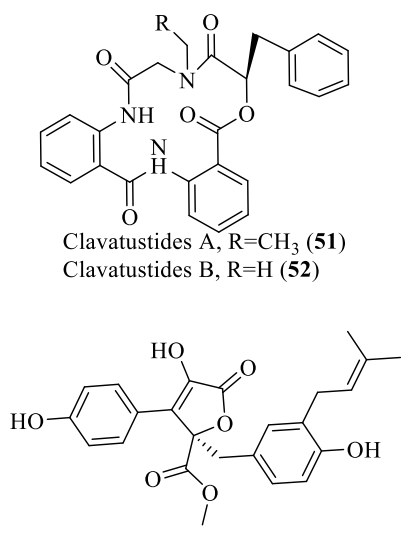

Aspergilamide $\mathrm{C}, \mathrm{R}=\mathrm{CH}\left(\mathrm{CH}_{3}\right) \mathrm{CH}_{2} \mathrm{CH}_{3}(\mathbf{5 3})$

$\mathrm{HO}^{-}$<smiles>[R]C(NC(C)=O)C(=O)N(C)C1Cc2ccc(O)cc2C(/C=C/c2c[nH]c3ccccc23)C1=O</smiles>

Aspergilamide D, $\mathrm{R}=\mathrm{CH}\left(\mathrm{CH}_{3}\right) \mathrm{CH}_{2} \mathrm{CH}_{3}$ (54)

Butyrolactone I (55)<smiles>CC(C)CC(NC(=O)C(Cc1ccccc1C(=O)O)N(C)C(=O)C(N)C(C)C)C(=O)O</smiles>

Simplicilliumtide A (56)<smiles>CC(C)C(C(=O)Nc1ccccc1C(=O)O)C(=O)C1Nc2ccccc2CC1N(C)C(=O)[C@@H](N)C(C)C</smiles>

Simplicilliumtide B (57)

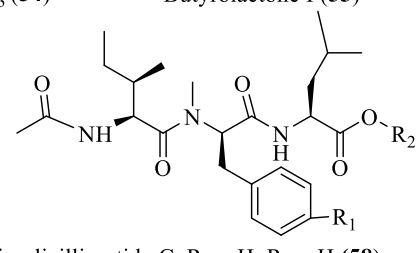

Simplicilliumtide $C, R_{1}=H, R_{2}=H(\mathbf{5 8})$

Simplicilliumtide D, $\mathrm{R}_{1}=\mathrm{OH}, \mathrm{R}_{2}=\mathrm{H}$ (59)

Simplicilliumtide E, $\mathrm{R}_{1}=\mathrm{OH}, \mathrm{R}_{2}=\mathrm{CH}_{3}(\mathbf{6 0})$<smiles>CC(=O)N[C@@H](C(=O)O)C(=O)N(C)C(Cc1ccccc1)C(=O)N(C(C)C)C(C)C</smiles>

Simplicilliumtide F (61)

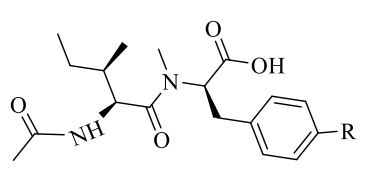

Simplicilliumtide $\mathrm{G}, \mathrm{R}=\mathrm{OH}(\mathbf{6 2})$ Simplicilliumtide H, R = H (63)

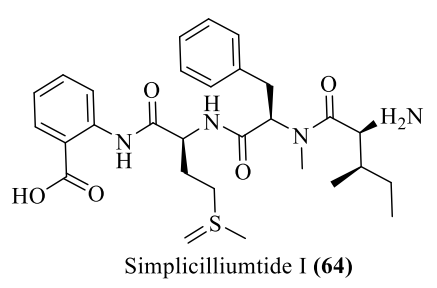

$\underset{\mathrm{H}}{\mathrm{O}}$

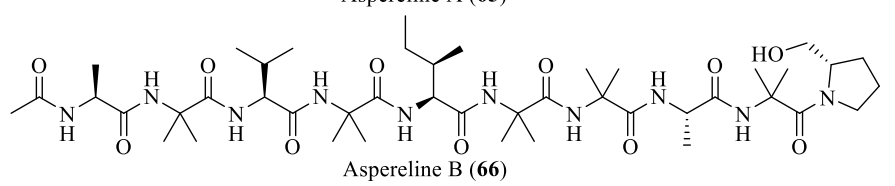

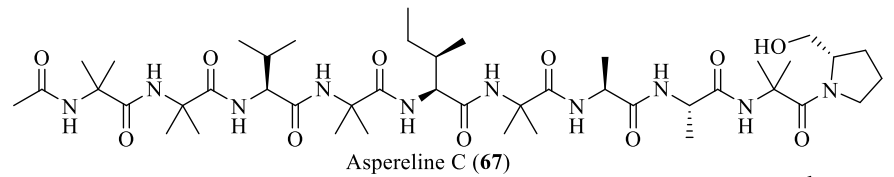

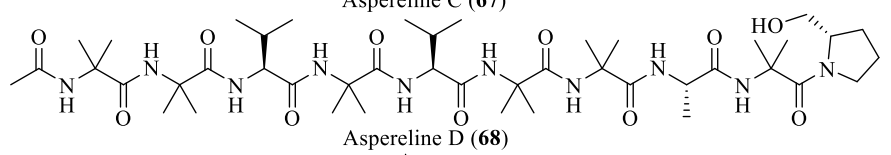

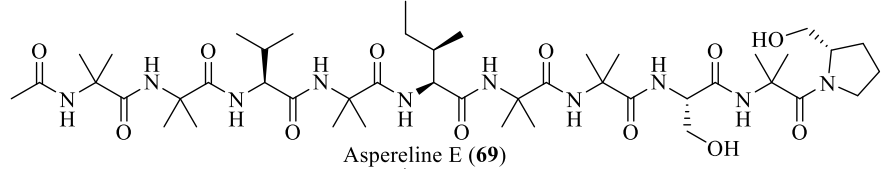

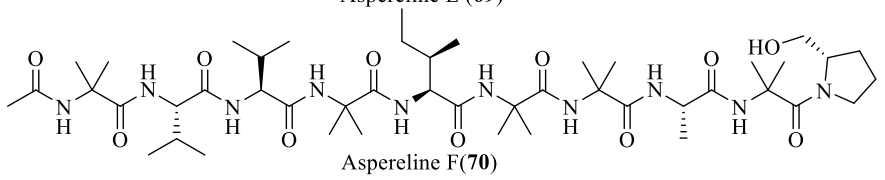

Figure 3. Bioactive polypeptides isolated from deep-sea fungi. 


\subsection{Ester and Phenolic Derivatives}

Six new ester derivatives (71-76; Figure 4) were extracted from Aspergillus ungui NKH-007 and showed inhibition of sterol O-acyltransferase (SOAT) enzymes in Chinese hamster ovary (CHO) cells and are thus considered to be good candidates for an anti-atherosclerotic agent [32]. Five new phenolic compounds (77-81; Figure 4) isolated from Penicillium sp. and Aspergillus versicolor showed potent activity against Staphylococcus aureus and Bacillus subtilis, with MIC values of $2-8 \mu \mathrm{g} / \mathrm{mL}[33,34]$. However, compounds $\mathbf{7 8 - 8 1}$ expressed antiviral activity toward HSV-1, with $\mathrm{EC}_{50}$ values of $3.12-6.25 \mu \mathrm{M}[34]$.<smiles>[R3]c1c([R])c([R3])c2c(c1[R3])Oc1c(C)c(O)c([Y9])c(/C(C)=C/C)c1O2</smiles>

7-Chlorofolipastatin, $\mathrm{R}_{1}=\mathrm{CH}\left(\mathrm{CH}_{3}\right) \mathrm{CH}\left(\mathrm{CH}_{3}\right), \mathrm{R}_{2}=\mathrm{H}, \mathrm{R}_{3}=\mathrm{CH}_{3}, \mathrm{R}_{4}=\mathrm{Cl}$ (71)

Folipostatin, $\mathrm{R}_{1}=\mathrm{CH}\left(\mathrm{CH}_{3}\right) \mathrm{CH}\left(\mathrm{CH}_{3}\right), \mathrm{R}_{2}=\mathrm{H}, \mathrm{R}_{3}=\mathrm{CH}_{3}, \mathrm{R}_{4}=\mathrm{H}$ (72)

Unguinol, $\mathrm{R}_{1}=\mathrm{CH}_{3}, \mathrm{R}_{2}=\mathrm{H}, \mathrm{R}_{3}=\mathrm{CH}_{3}, \mathrm{R}_{4}=\mathrm{H}$ (73)

2-Chlorounginol, $\mathrm{R}_{1}=\mathrm{CH}_{3}, \mathrm{R}_{2}=\mathrm{Cl}, \mathrm{R}_{3}=\mathrm{CH}_{3}, \mathrm{R}_{4}=\mathrm{H}$ (74)

2,7-Dichloroung, $\mathrm{R}_{1}=\mathrm{CH}_{3}, \mathrm{R}_{2}=\mathrm{Cl}, \mathrm{R}_{3}=\mathrm{CH}_{3}, \mathrm{R}_{4}=\mathrm{Cl}$ (75)

Nornidulin, $\mathrm{CH}_{3}, \mathrm{R} 1=\mathrm{CH}_{3}, \mathrm{R}_{2}=\mathrm{Cl}, \mathrm{R}_{3}=\mathrm{Cl}, \mathrm{R}_{4}=\mathrm{Cl}$ (76)<smiles>CC(=O)CCCC(c1c(C)cc(Oc2cc(C)cc(O)c2)cc1O)c1c(-c2ccccc2)cc2c(c1O)C(=O)c1c(O)cc(O)cc1C2=O</smiles>

Aspergilol H (79)

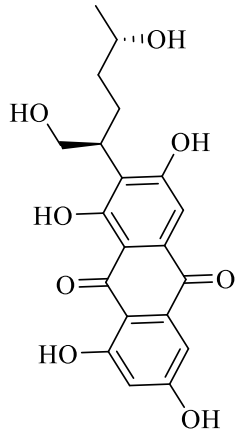

Aspergilol I (80)<smiles>CC(=O)c1c(C)cc(O)c(CC=C(C)C)c1O</smiles>

Pestalotionol (77)<smiles>CC(=O)OCC(CCc1c(O)cc2c(c1O)C(=O)c1c(O)cc(O)cc1C2=O)C(C)=O</smiles>

Coccoquinone A (81)

Figure 4. Ester and phenolic derivatives obtained from deep-sea fungi.

\subsection{Piperazine Derivatives}

Fourteen new piperazine derivatives (82-95; Figure 5) reported from marine fungi during the last five years. These derivatives were isolated from genera of Penicillium, Aspergillus, and Dichotomomyces collected from deep-sea sediments. Compounds 82-84 showed strong cytotoxicity with $\mathrm{IC}_{50}$ of 1.7 and $2 \mu \mathrm{M}$ against K562 and mouse lymphoma cell line, respectively; similarly, compounds 91-95 also showed strong cytotoxic activity [35-37]. Compounds 85-89 showed antibacterial activity against Staphylococcus aureus with the MIC values of $6.25-12.5 \mu \mathrm{g} / \mathrm{mL}$ [21]. The new compound 90 also showed stronger inhibition activity against $\alpha$-glucosidase with $\mathrm{IC}_{50}$ value of $138 \mu \mathrm{M}$ [37]. 
<smiles>CSC1C(=O)N(C)C(=Cc2ccc(OCC=C(C)C)cc2)C(=O)N1C</smiles>

Fusaperazine F (82)

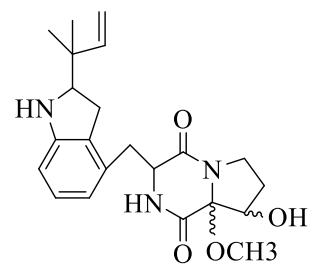

8-Hydroxy-brevianamide R (86)

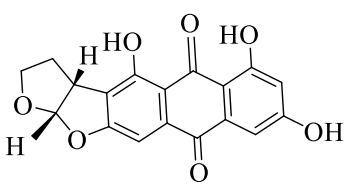

Versicolorin (89)

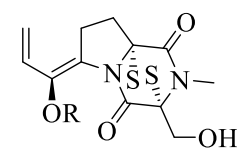

Gliotoxin, $\mathrm{R}=\mathrm{H}$ (92)

Acetylgliotoxin, $\mathrm{R}=\mathrm{Ac}(\mathbf{9 3})$

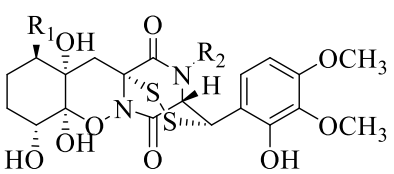

Pretrichodermamide $\mathrm{C}$, $\mathrm{R}_{1}=\mathrm{OH}, \mathrm{R}_{2}=\mathrm{CH}_{3}(\mathbf{8 3})$ N-methylpretrichodermamide $\mathrm{B}$, $\mathrm{R}_{1}=\mathrm{Cl}, \mathrm{R}_{2}=\mathrm{CH}_{3}$ (84)

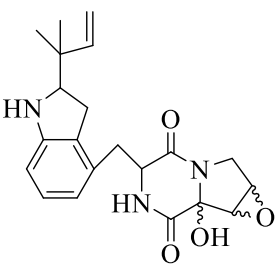

7,8-Epoxy-brevianamide Q (85)

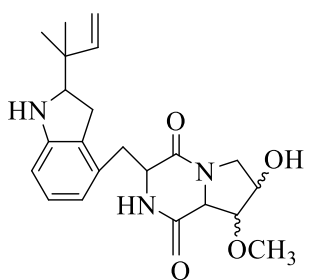

8-Epoxy-brevianamide R (87)

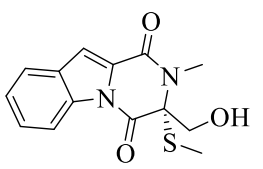

Dichotocejpin A (90)

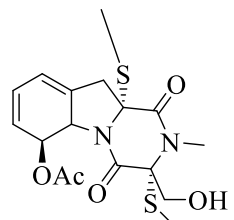

6-Acetylbis(methylthio)gliotoxin(94)

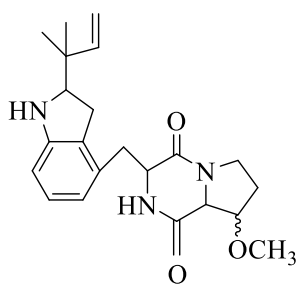

Brevianamide R (88)

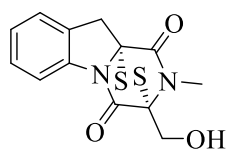

6-Deoxy-5a,6-didehydrogliotoxin (91)

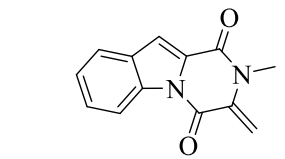

1,2,3,4-Tetrahydro-2-methyl

-3-methylene-1,4-

dioxopyrazino[1,2-a]indol (95)

Figure 5. Piperazine derivatives isolated from deep-sea fungi.

\subsection{Terpenoid Compounds}

Thirty-six new and important bioactive terpenoids (96-131; Figure 6) have been isolated from marine fungi extracted from the deep-sea sediments since 2013. Compounds 96-113 were isolated from Penicillium spp., while compounds 114-131 were extracted from Aspergillus spp. Breviones (96-99), isolated from the deepest sediment-derived fungus Penicillium sp. (5115 m depth), displayed diverse activities, such as cytotoxicity against HeLa, MCF-7, and A549 cells with $\mathrm{IC}_{50}$ values of 7.44 to $32.5 \mu \mathrm{M}$, respectively, and growth inhibition of HIV-1 with $\mathrm{EC}_{50}$ value of $14.7 \mu \mathrm{M}$ against C8166 cells [22,38]. Compounds 100-110 showed antibiotic and inhibition activities against silkworm, while 20-nor-isopimarane diterpenoids, including aspewentins (114-118), asperethers (121-125), asperoloids (119-120), and compounds 130 and 131, showed cytotoxic activities [33,39-45]. However, the spirocyclic diterpenes (111-113) exhibited strong anti-allergic effect with $18 \%$ inhibition at $20 \mu \mathrm{g} / \mathrm{mL}$ [46]. Interestingly, four new compounds (126-129) were extracted from hydrothermal vent-derived Aspergillus sydowii, through activation of a new pathway for secondary metabolite production by the addition of a 5-azacytidine (a DNA methyltransferase inhibitor). These compounds showed anti-inflammatory and antidiabetic activities and are thus the first secondary metabolites isolated from fungi which have both antidiabetic and anti-inflammatory activities [47]. 


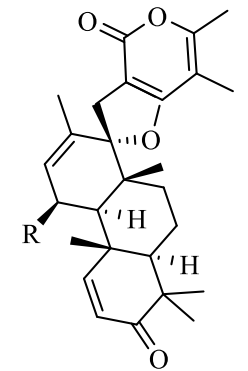

Brevione $\mathrm{F}, \mathrm{R}=\mathrm{H}(\mathbf{9 6})$ Brevione I, $\mathrm{R}=\mathrm{OH}$ (97) Brevione $\mathrm{H}$ (98)

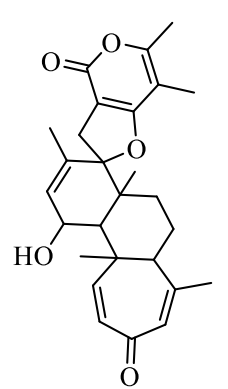

Brevione G (99)

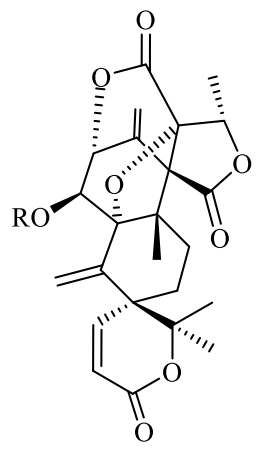

Dehydroaustin $\mathrm{R}=\mathrm{Ac}(\mathbf{1 0 0})$

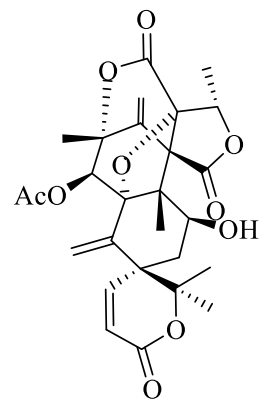

Dehydroaustinol R=H (101) 7-Hydroxydehydroaustin (102)

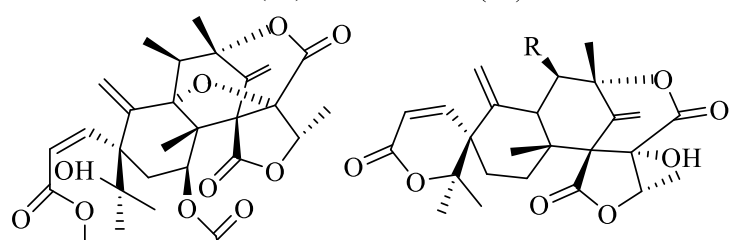

Austinol, $\mathrm{R}=\mathrm{OH}$ (104) Austin, $\mathrm{R}=\mathrm{OAc}(\mathbf{1 0 5})$

Austinolide, $\mathrm{R}=\mathrm{H}$ (106)

Austinone (103)<smiles>CC(=O)O[C@H]1C=C(Cl)C2=CC(=O)C(O)=C[C@@]2(C)[C@H]1C</smiles>

1-Chloro-3ß-acetoxy-7-hydroxytrinoreremophil-1,6,9-trien-8-one(107)<smiles>CO[C@]12C=C3[C@@H](Cl)[C@H](O)[C@@H](OC(C)=O)[C@H](C)[C@]3(C)CC13OC(C)(CO2)O3</smiles>

Eremophilane-type sesquiterpene (108)<smiles>CC(=O)OC1C2OC2C2=CC(=O)C3(CO)OC2(C)C13C</smiles><smiles>C=C(C)[C@H]1CC2(C)C(=CC1=O)[C@H](O)[C@H](O)[C@H](O)C2C</smiles>
Guignarderemophilane F (110)

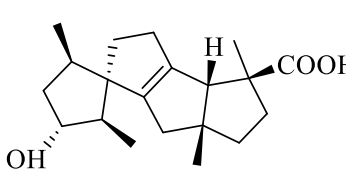

Spirograterpene A (111)

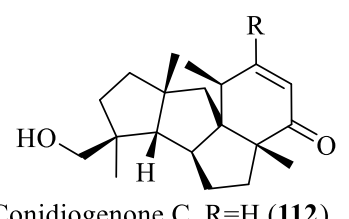

Conidiogenone $\mathrm{C}, \mathrm{R}=\mathrm{H}$ (112) Conidiogenone $\mathrm{I}, \mathrm{R}=\mathrm{H}(\mathbf{1 1 3})$<smiles>C=CC1(C)CCc2c3c(cc(O)c2C1O)C(C)(C)[C@@H](O)CC3</smiles><smiles>C=CC1(C)CCC2=C(C1)C(=O)C=CC1(O)CCCC(C)(C)C21</smiles><smiles>C=CC1(C)CCC2=C(C1)C(=O)C=C1C3C(C)(C)CCC(O)C123</smiles><smiles>C=CC1(C)CCC2(O)C3=C(C=CC2(C)C1)C(=O)CCC3(C)C</smiles><smiles>C=CC1(C)CCc2c(cc(O)c3c2CCC(O)C3(C)C)C1(C)C</smiles>

Aspewentin A (114) Aspewentins D (115)

Aspewentins F (116)

Aspewentin G (117)

Aspewentin H (118)

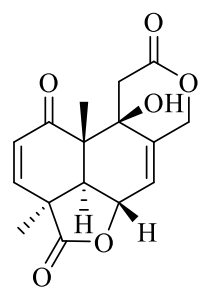

Asperolides D (119) Asperolides E

(120)

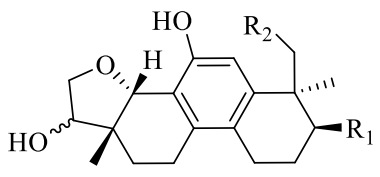

Asperethers $\mathrm{A}, \mathrm{R}_{1}=\mathrm{OH}, \mathrm{R}_{2}=\mathrm{H}$ (121)

Asperethers $B, R_{1}=H, R_{2}=H$ (122)

Asperethers $\mathrm{C}, \mathrm{R}_{1}=\mathrm{H}, \mathrm{R}_{2}=\mathrm{OH}$ (123)

Asperethers $\mathrm{D}, \mathrm{R}_{1}=\mathrm{OH}, \mathrm{R}_{2}=\mathrm{OAc}(\mathbf{1 2 4})$

Asperethers $\mathrm{E}, \mathrm{R}_{1}=\mathrm{OH}, \mathrm{R}_{2}=\mathrm{H}$ (125)<smiles>[R3]CC(C)CCCC([R3])(C)C1CCC([B])CC1O</smiles>

(7S)-(+)-7-O-methylsydonol, $\mathrm{R}_{1}=\mathrm{OMe}, \mathrm{R}_{2}=\mathrm{H}, \mathrm{R}_{3}=\mathrm{CH}_{2} \mathrm{OH}$ (126) (7S,11S)-(+)-12-hydroxysydonic acid, $\mathrm{R}_{1}=\mathrm{OH}, \mathrm{R}_{2}=\mathrm{OH}, \mathrm{R}_{3}=\mathrm{COOH}(\mathbf{1 2 7})$ 7-deoxy-7,14-didehydrosydonol, $\mathrm{R}_{1}=\mathrm{OH}, \mathrm{R}_{2}=\mathrm{H}, \mathrm{R}_{3}=\mathrm{CH}_{2} \mathrm{OH}(\mathbf{1 2 8})$<smiles>C=C(CCCCC)c1ccc(CO)cc1O</smiles>

(S)-(+)-Sydonol (129)

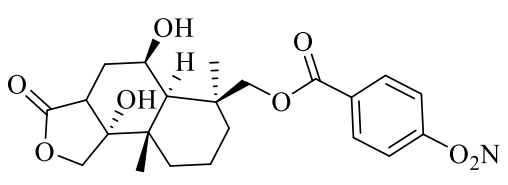

6b, 9a-Dihydroxy-14-p-nitrobenzoylcinnamolide (130)

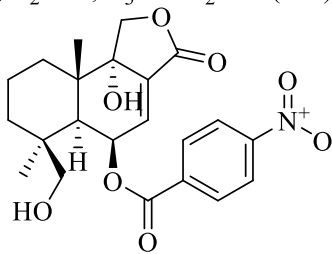

Insulicolide A (131)

Figure 6. Structures of terpenoid secondary metabolites obtained from deep-sea fungi.

\subsection{Other Unrelated Compounds}

Twenty secondary metabolites with different structures were isolated from deep-sea fungi, mainly from Penicillium spp. and Aspergillus spp. (132-151; Figure 7). Penipacids A-F (134-139), 
polyoxygenated sterol (132), dicitrinone B (133) and butanolide A (140), which were isolated from deep-sea sediments-derived Penicillium spp., showed cytotoxic activities against RKO, MCF-7, PTP1B and A375 cancer cell lines with $\mathrm{IC}_{50}$ values of 8.4-28.4 $\mu \mathrm{M}[38,42,48,49]$. Similarly, four isocoumarins, penicillisocoumarin A-D (147-150), and an isocoumarins aspergillumarin B (151) were also isolated from Penicillium which showed weak antibacterial activities [33]. Four antibiotic cyclopenin derivatives compounds (141-144) and a series of antitumor wentilactones $(\mathbf{1 4 5 , 1 4 6 )}$ were isolated from Aspergillus spp. [50,51].

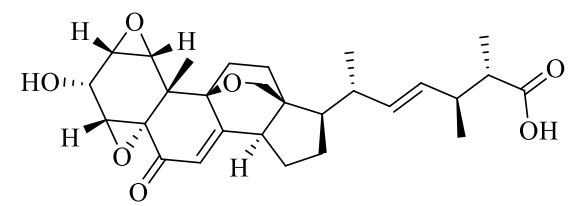

Sterolic acid (132)<smiles>CC1C(=O)C=C2C(=COC(C)[C@@H]2C)C(O)=c2c(O)c3c(c(O)c2C1=O)=COC(C)[C@@H]3C</smiles>

Dicitrinone B (133)<smiles>[R]C(C)(C)CN=C(C)Nc1ccccc1C(=O)O</smiles>

Penipacid A, $\mathrm{R}=\mathrm{OH}$ (134) Penipacid B, R=OMe (135)<smiles>[R]OC(=O)/N=C(\C)Nc1ccccc1C(=O)O</smiles>

Penipacid $\mathrm{C}, \mathrm{R}=\mathrm{H}(\mathbf{1 3 6})$ Penipacid D R=Me (137)<smiles>O=C(O)c1ccccc1N/C=N/c1ccco1</smiles>

Penipacid E (138)<smiles>CC(=O)C(C)=Nc1ccccc1C(=O)O</smiles>

Penipacid F (139)<smiles>CC[C@H](O)CC/C=C\C=C\[C@@H](O)[C@H]1CCC(=O)O1</smiles>

Butanolide A (140)<smiles>COc1ccc2c(c1)C(=O)N(C)[C@@H](Cc1ccccc1)C(=O)N2</smiles><smiles>COc1ccc2c(c1)C(=O)N(C)/C(=C\c1ccccc1)C(=O)N2</smiles>

7-Methoxydehydrocyclopeptin (142)<smiles>COc1c(-c2ccccc2)c2cccc(O)c2[nH]c1=O</smiles>

9-Hydroxy-3-methoxyviridicatin (144)<smiles>[R]c1ccc(O)c2c1C[C@@H](CCCC(C)=O)OC2=O</smiles>

Penicillisocoumarin A, $\mathrm{R}=\mathrm{OH}(\mathbf{1 4 7})$ Penicillisocoumarin $\mathrm{B}, \mathrm{R}=\mathrm{H}$ (148)

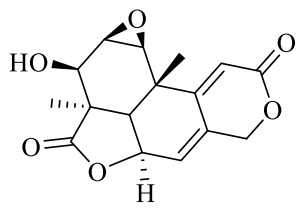

Wentilactone A (145)<smiles>[R]c1ccc(O)c2c1C[C@H](CCCO)OC2=O</smiles>

Penicillisocoumarin C (149)<smiles>COc1ccc2c(c1)C(=O)N(C)C1(OC1c1ccccc1)C(=O)N2</smiles>

7-Methoxycyclopenin (143)<smiles>C[C@@]12COC(=O)C=C1C=C[C@H]1OC(=O)[C@@]1(C)C[C@@H](O)C2</smiles>

Wentilactone B (146)<smiles>[R]c1ccc(O)c2c1C[C@H](CCC[C@H](C)O)OC2=O</smiles>

Penicillisocoumarin $\mathrm{D}, \mathrm{R}=\mathrm{OH}(\mathbf{1 5 0})$ Aspergillumarins $\mathrm{B}, \mathrm{R}=\mathrm{H}(\mathbf{1 5 1})$

Figure 7. Bioactive metabolites derived from deep-sea fungi. 
Table 1. Secondary metabolites extracted from deep-sea fungi during 2013-2019.

\begin{tabular}{|c|c|c|c|c|c|c|}
\hline Metabolites & Fungal Species & Source & Location & Depth (m) ${ }^{*}$ & Bioactivity & Ref. \\
\hline \multicolumn{7}{|c|}{ Polyketide } \\
\hline Methyl-isoverrucosidinol (1) & Penicillium sp. Y-50-10 & Sulfur-rich Sediment & hydrothermal vent, Taiwan & - & Antibiotic & [13] \\
\hline Penilactone A (2) & Penicillium crustosum PRB-2 & Sediment & Prydz Bay, Antarctica & 526 & NF-kB inhibition & [14] \\
\hline $\begin{array}{l}\text { Aspiketolactonol (3) } \\
\text { Aspilactonols A-F (4-9) } \\
\text { Aspyronol (10) } \\
\text { Epiaspinonediol (11) }\end{array}$ & Aspergillus sp. 16-02-1 & Hydrothermal vent water & Lau Basin, Southwest Pacific Ocean, & 2255 & Cytotoxic & [15] \\
\hline $\begin{array}{l}\text { Ascomycotin A (12) } \\
\text { Diorcinol (13) }\end{array}$ & Ascomycota sp. Ind19F07 & Sediment & Indian Ocean & 3614 & Antibiotic & [16] \\
\hline Engyodontiumones A-J (14-22) & Engyodontium album DFFSCS021 & Sediment & South China Sea & 3739 & Cytotoxic & [18] \\
\hline $\begin{array}{l}\text { Lindgomycin (23) } \\
\text { Ascosetin (24) }\end{array}$ & Lindgomycetaceae strains KF970 and LF327 & Sediment & Greenland Sea, Baltic Sea & 3650 & Antibiotic & [17] \\
\hline \multicolumn{7}{|c|}{ Nitrogen-containing compounds } \\
\hline Brevicompanines D-H (25-29) & Penicillium sp. F1 & Sediment & - & 5080 & $\begin{array}{l}\text { LPS-induced } \\
\text { inflammation }\end{array}$ & [22] \\
\hline Cyclopiamide B-J (30-38) & Penicillium commune DFFSCS026 & Sediment & South China Sea & 3563 & Cytotoxic & [24] \\
\hline $\begin{array}{l}\text { Penipanoid A (39) } \\
\text { Quinazolinone (40) }\end{array}$ & Penicillium paneum SD-44 & Sediment & South China Sea & 201 & Cytotoxic & [23] \\
\hline ( \pm ) Brevianamide R (41) & $\begin{array}{l}\text { Aspergillus } \\
\text { versicolor MF180151 }\end{array}$ & Sediment & Bohai Sea, China & - & Antibacterial & [21] \\
\hline Circumdatin F and G (42-43) & Aspergillus westerdijkiae SCSIO 05233 & Sediment & South China Sea & 4593 & Cytotoxic & [20] \\
\hline $\begin{array}{l}\text { Oximoaspergillimide (44) } \\
\text { Neohydroxyaspergillic (45) } \\
\text { Neoaspergillic (46) }\end{array}$ & Aspergillus sp. (CF07002) & Water & Pacific Ocean off the coast of Panama & & $\begin{array}{l}\text { Cytotoxic } \\
\text { Antibiotic }\end{array}$ & [19] \\
\hline Varioxepine A (47) & Paecilomyces variotii EN-291 & Deep sea water & - & - & Antibiotic & [26] \\
\hline Neoechinulin A (48) & Microsporum sp. (MFS-YL) & Red alga & Guryongpo, Korea & - & Cytotoxic & [25] \\
\hline \multicolumn{7}{|c|}{ Polypeptide } \\
\hline Canescenin A and B (49-50) & Penicillium canescens SCSIO z053 & Water & East China Sea & 2013 & Antibacterial & [27] \\
\hline Clavatustide A and B (51-52) & Aspergillus clavatus $\mathrm{C} 2 \mathrm{WU}$ & Hydrothermal vent crab & Taiwan Kueishantao & - & Cytotoxic & [29] \\
\hline $\begin{array}{l}\text { Aspergillamides C and D (53-54) } \\
\text { Butyrolactone I (55) }\end{array}$ & Aspergillus terreus SCSIO 41008 & Sponge & Guangdong, China & - & $\begin{array}{l}\text { Cytotoxic } \\
\text { Antibiotic }\end{array}$ & [30] \\
\hline Simplicilliumtides A-I (56-64) & Simplicillium obclavatum EIODSF 020 & Sediment & East Indian Ocean & 4571 & Cytotoxic & [31] \\
\hline Asperelines A-F (65-70) & Trichoderma asperellum & Sediment & Antarctic Penguin Island & _- & Antibiotic & [28] \\
\hline
\end{tabular}


Table 1. Cont

\begin{tabular}{|c|c|c|c|c|c|c|}
\hline Metabolites & Fungal Species & Source & Location & Depth $(\mathrm{m})^{*}$ & Bioactivity & Ref. \\
\hline \multicolumn{7}{|c|}{ Esters } \\
\hline $\begin{array}{l}\text { 7-chlorofolipastatin (71) } \\
\text { Folipostatin B (72) } \\
\text { Unguinol (73) } \\
\text { 2-chlorounginol (74) } \\
\text { 2,7-dichlorounguinol (75) } \\
\text { Nornidulin (76) }\end{array}$ & Aspergillus ungui NKH-007 & Sediment & Suruga Bay, Japan & - & $\begin{array}{l}\text { Anti-atherosclerotic } \\
\text { Cytotoxic } \\
\text { Antibiotic }\end{array}$ & {$[32]$} \\
\hline \multicolumn{7}{|c|}{ Phenolic } \\
\hline Pestalotionol (77) & Penicillium sp. Y-5-2 & Hydrothermal vent water & Kueishantao off Taiwan & - & Antibiotic & [33] \\
\hline $\begin{array}{l}\text { Aspergilol G-I (78-80) } \\
\text { Coccoquinone A (81) }\end{array}$ & Aspergillus versicolor SCSIO 41502 & Sediment & South China Sea & 2326 & $\begin{array}{l}\text { Anti-HSV-1 } \\
\text { Antioxidant } \\
\text { Antifouling }\end{array}$ & {$[34]$} \\
\hline \multicolumn{7}{|c|}{ Piperazine } \\
\hline Fusaperazine F (82) & Penicillium crustosum HDN153086 & Sediment & Prydz Bay, Antarctica & - & Cytotoxic & [35] \\
\hline $\begin{array}{l}\text { N-methyl-pretrichodermamide B (83) } \\
\text { Pretrichodermamide C (84) }\end{array}$ & Penicillium sp. (WN-11-1-3-1-2) & Hypersaline sediment & Wadi El-Natrun, Egypt & - & Cytotoxic & [36] \\
\hline $\begin{array}{l}\text { ( } \pm \text { ) 7,8-epoxy-brevianamide Q (85) } \\
\text { ( } \pm \text { 8-hydroxy-brevianamide R (86) } \\
\text { ( } \pm \text { ) 8-epihydroxy-brevianamide R (87) } \\
\text { Brevianamide R (88) } \\
\text { Versicolorin B (89) }\end{array}$ & Aspergillus versicolor MF180151 & Sediment & Bohai Sea, China & - & Antibiotic & {$[21]$} \\
\hline $\begin{array}{l}\text { Dichotocejpins A (90) } \\
\text { 6-deoxy-5a,6-didehydrogliotoxin (91) } \\
\text { Gliotoxin (92) } \\
\text { Acetylgliotoxin (93) } \\
\text { 6-acetylbis(methylthio)-gliotoxin (94) } \\
\text { 1,2,3,4-tetrahydro-2-methyl-3-methylene- } \\
\text { 1,4-dioxopyrazino [1,2-a] indole (95) }\end{array}$ & Dichotomomyces cejpii FS110 & Sediment & South China Sea & 3941 & $\begin{array}{l}\alpha \text {-Glucosidase } \\
\text { inhibition } \\
\text { Cytotoxic }\end{array}$ & [37] \\
\hline \multicolumn{7}{|c|}{ Terpenoid } \\
\hline Brevione F-I (96-99) & Penicillium sp. (MCCC 3A00005) & Sediment & Pacific Ocean & 5115 & $\begin{array}{l}\text { Cytotoxic } \\
\text { HIV-1 inhibition }\end{array}$ & {$[22,38]$} \\
\hline $\begin{array}{l}\text { Dehydroaustin (100) } \\
\text { Dehydroaustinol (101) } \\
\text { 7-hydroxydehydroaustin (102) } \\
\text { Austinone (103) } \\
\text { Austinol (104) } \\
\text { Austin (105) } \\
\text { Austinolide (106) }\end{array}$ & Penicillium sp. Y-5-2 & Hydrothermal vent water & Kueishantao off Taiwan & 8 & $\begin{array}{l}\text { Antibacterial } \\
\text { Anti-insectal }\end{array}$ & [33] \\
\hline
\end{tabular}


Table 1. Cont.

\begin{tabular}{|c|c|c|c|c|c|c|}
\hline Metabolites & Fungal Species & Source & Location & Depth $(\mathrm{m})^{*}$ & Bioactivity & Ref. \\
\hline $\begin{array}{l}\text { 1-chloro-3 } \beta \text {-acetoxy-7- } \\
\text { hydroxytrinoreremophil-1,6,9-trien- } \\
\text { 8-one (107) } \\
\text { Eremophilane-type sesquiterpenes (108) } \\
\text { Eremofortine C (109) }\end{array}$ & Penicillium sp. PR19N-1 & Sediment & Prydz Bay, Antarctica & 526 & Cytotoxic & {$[40,41]$} \\
\hline Guignarderemophilane F (110) & Penicillium sp. S-1-18 & Sediment & Antarctic & 1393 & Antibacterial & [42] \\
\hline $\begin{array}{l}\text { Spirograterpene A (111) } \\
\text { Conidiogenone C and I (112-113) }\end{array}$ & Penicillium granulatum MCCC 3A00475 & Water & Prydz Bay of Antarctica & 2284 & Antiallergic & [46] \\
\hline $\begin{array}{l}\text { Aspewentin A and D-H (114-118) } \\
\text { Asperethers A-E (121-125) } \\
\text { Asperolides D and E (119-120) }\end{array}$ & Aspergillus wentii SD-310 & Sediment & South China Sea & 2038 & $\begin{array}{l}\text { Antimicrobial } \\
\text { Cytotoxic } \\
\text { Anti-inflammatory }\end{array}$ & {$[39,43,44]$} \\
\hline $\begin{array}{l}\text { (7S)-(+)7-O-methylsydonol (126) } \\
\text { (7S,11S)-(+)-12-hydroxysydonic acid (127) } \\
\text { 7-deoxy-7,14-didehydrosydonol (128) } \\
\text { (S)-(+)-sydonol (129) }\end{array}$ & Aspergillus sydowii & Sediment & Hsinchu, Taiwan & - & Anti-inflammatory & [47] \\
\hline $\begin{array}{l}\text { 6b,9a-dihydroxy-14-p- } \\
\text { nitrobenzoylcinnamolide (130) } \\
\text { Insulicolide A (131) }\end{array}$ & Aspergillus ochraceus Jcma1F17 & Marine alga Coelarthrum sp. & South China Sea & - & $\begin{array}{l}\text { Antiviral } \\
\text { Cytotoxic }\end{array}$ & [45] \\
\hline \multicolumn{7}{|c|}{ Other compounds } \\
\hline Sterolic acid (132) & Penicillium sp. MCCC 3A00005 & Sediment & East Pacific Ocean & 5115 & Cytotoxic & {$[38]$} \\
\hline Dicitrinone B (133) & Penicillium citrinum & Sediment & Langqi Island, Fujian, China & - & Antitumor & [49] \\
\hline Penipacids A-F (134-139) & Penicillium paneum SD-44 & Sediment & South China Sea & - & Cytotoxic & [48] \\
\hline Butanolide A (140) & Penicillium sp. S-1-18 & Sediment & Antarctic seabed & 1393 & Cytotoxic & [42] \\
\hline $\begin{array}{l}\text { 7-Methoxycyclopeptin (141) } \\
\text { 7-Methoxy dehydro cyclopeptin (142) } \\
\text { 7-Methoxy cyclopenin (143) } \\
\text { 9-Hydroxy-3-methoxyviridicatin (144) }\end{array}$ & Aspergillus versicolor XZ-4 & Hydrothermal vent crab & Kueishantao, Taiwan & & Antibiotic & {$[50]$} \\
\hline Wentilactone A and B (145-146) & Aspergillus dimorphicus SD317 & Sediment & South China Sea & 2038 & Antitumor & [51] \\
\hline $\begin{array}{l}\text { Penicillisocoumarin A-D (147-150) } \\
\text { Aspergillumarins B (151) }\end{array}$ & Penicillium sp. Y-5-2 & Hydrothermal vent water & Kueishantao off Taiwan & 8 & Antibacterial & [33] \\
\hline
\end{tabular}

* Depth represents water depth below the surface. 


\section{Conclusions and Perspective}

The results of current studies indicate that the deep-sea extreme environmental fungi are one of the rich and unexploited sources of important medicinal lead compounds. Most of the fungi (e.g., Penicillium spp. and Aspergillus spp.) living in the extreme environments of the deep-sea have the potential to synthesize new bioactive compounds. However, the research on deep-sea fungi and their metabolites is very limited due to the difficulty of sampling and the limitation of culture technology. Thanks to the advances in genome technology and the implementation of the deep-sea drilling program, novel compounds with great biological activities are expected from these fungi in the near future. From the literature review, we can say these fungi from the extreme environments have the potential to produce clinically important natural products. The compounds we discussed in this review show strong bioactivities and might have the potential to be a future anticancer drug. Among them, terpenoid derivatives were the most important and abundant compound category which were mainly isolated from deep-sea derived Penicillium spp. and Aspergillus spp. This class of compounds showed strongest antibiotic and cytotoxic activities as compared to other classes of compounds and has the potential to be a future candidate for anticancer drugs, especially brevione, which was isolated from the deepest part of the sea and showed the strongest cytotoxic activity.

Author Contributions: Writing—original draft preparation, M.Z.u.A.; writing—review and editing, Y.-N.M., Y.-R.X. and C.-H.L. All authors have read and agreed to the published version of the manuscript.

Funding: This work was financially supported by the National Natural Science Foundation of China (General Program: 41773083, 41973073; Major Program: 91951121).

Conflicts of Interest: The authors declare that they have no competing interests.

\section{References}

1. König, G.M.; Kehraus, S.; Seibert, S.F.; Abdel-Lateff, A.; Müller, D. Natural products from marine organisms and their associated microbes. Chem. Bio. Chem. 2006, 7, 229-238. [CrossRef] [PubMed]

2. Chen, G.; Wang, H.-F.; Pei, Y.-H. Secondary metabolites from marine-derived microorganisms. J. Asian Nat. Prod. Res. 2014, 16, 105-122. [CrossRef] [PubMed]

3. Agrawal, S.; Adholeya, A.; Deshmukh, S.K. The pharmacological potential of non-ribosomal peptides from marine sponge and tunicates. Front. Pharmacol. 2016, 7, 333. [CrossRef] [PubMed]

4. Deshmukh, S.K.; Prakash, V.; Ranjan, N. Recent advances in the discovery of bioactive metabolites from Pestalotiopsis. Phytochem. Rev. 2017, 16, 883-920. [CrossRef]

5. Deshmukh, S.K.; Prakash, V.; Ranjan, N. Marine fungi: A source of potential anticancer compounds. Front. Microbiol. 2018, 8, 2536. [CrossRef]

6. Danovaro, R.; Corinaldesi, C.; Dell'Anno, A.; Snelgrove, P.V. The deep-sea under global change. Curr. Biol. 2017, 27, R461-R465. [CrossRef]

7. Barone, G.; Varrella, S.; Tangherlini, M.; Rastelli, E.; Dell'Anno, A.; Danovaro, R.; Corinaldesi, C. Marine fungi: Biotechnological perspectives from deep-hypersaline anoxic basins. Diversity 2019, 11, 113. [CrossRef]

8. Hamilton-Miller, J. Development of the semi-synthetic penicillins and cephalosporins. Int. J. Antimicrob. 2008, 31, 189-192. [CrossRef]

9. Carroll, A.R.; Copp, B.R.; Davis, R.A.; Keyzers, R.A.; Prinsep, M.R. Marine natural products. Nat. Prod. Rep. 2019, 36, 122-173. [CrossRef]

10. Wang, Y.-T.; Xue, Y.-R.; Liu, C.-H. A brief review of bioactive metabolites derived from deep-sea fungi. Mar. Drugs 2015, 13, 4594-4616. [CrossRef]

11. Arifeen, M.Z.U.; Liu, C.-H. Novel enzymes isolated from marine-derived fungi and its potential applications. United J. Biochem. Biotechnol. 2018, 1,1-11.

12. Arifeen, M.Z.U.; Xue, Y.-R.; Liu, C.-H. Deep-sea fungi: Diversity, enzymes, and bioactive metabolites. In Fungi in Extreme Environments: Ecological Role and Biotechnological Significance; Springer: Berlin, Germany, 2019; pp. 331-347. 
13. Pan, C.; Shi, Y.; Auckloo, B.; Chen, X.; Chen, C.-T.; Tao, X.; Wu, B. An unusual conformational isomer of verrucosidin backbone from a hydrothermal vent fungus, Penicillium sp. Y-50-10. Mar. Drugs 2016, 14, 156. [CrossRef]

14. Wu, G.; Ma, H.; Zhu, T.; Li, J.; Gu, Q.; Li, D. Penilactones A and B, two novel polyketides from Antarctic deep-sea derived fungus Penicillium crustosum PRB-2. Tetrahedron 2012, 68, 9745-9749. [CrossRef]

15. Chen, X.-W.; Li, C.-W.; Cui, C.-B.; Hua, W.; Zhu, T.-J.; Gu, Q.-Q. Nine new and five known polyketides derived from a deep sea-sourced Aspergillus sp. 16-02-1. Mar. Drugs 2014, 12, 3116-3137. [CrossRef]

16. Tian, Y.-Q.; Lin, X.-P.; Liu, J.; Kaliyaperumal, K.; Ai, W.; Ju, Z.-R.; Yang, B.; Wang, J.; Yang, X.-W.; Liu, Y. Ascomycotin A, a new citromycetin analogue produced by Ascomycota sp. Ind19F07 isolated from deep sea sediment. Nat. Prod. Res. 2015, 29, 820-826. [CrossRef]

17. Wu, B.; Wiese, J.; Labes, A.; Kramer, A.; Schmaljohann, R.; Imhoff, J. Lindgomycin, an unusual antibiotic polyketide from a marine fungus of the Lindgomycetaceae. Mar. Drugs 2015, 13, 4617-4632. [CrossRef]

18. Yao, Q.; Wang, J.; Zhang, X.; Nong, X.; Xu, X.; Qi, S. Cytotoxic polyketides from the deep-sea-derived fungus Engyodontium album DFFSCS021. Mar. Drugs 2014, 12, 5902-5915. [CrossRef]

19. Cardoso-Martínez, F.; de la Rosa, J.M.; Díaz-Marrero, A.R.; Darias, J.; D’Croz, L.; Cerella, C.; Diederich, M.; Cueto, M. Oximoaspergillimide, a fungal derivative from a marine isolate of Aspergillus sp. Eur. J. Org. Chem. 2015, 2015, 2256-2261. [CrossRef]

20. Fredimoses, M.; Zhou, X.; Ai, W.; Tian, X.; Yang, B.; Lin, X.; Xian, J.-Y.; Liu, Y. Westerdijkin A, a new hydroxyphenylacetic acid derivative from deep sea fungus Aspergillus westerdijkiae SCSIO 05233. Nat. Prod. Res. 2015, 29, 158-162. [CrossRef]

21. Hu, J.; Li, Z.; Gao, J.; He, H.; Dai, H.; Xia, X.; Liu, C.; Zhang, L.; Song, F. New diketopiperazines from a marine-derived fungus strain Aspergillus versicolor MF180151. Mar. Drugs 2019, 17, 262. [CrossRef]

22. Zhang, X.; Li, S.-J.; Li, J.-J.; Liang, Z.-Z.; Zhao, C.-Q. Novel natural products from extremophilic fungi. Mar. Drugs 2018, 16, 194. [CrossRef] [PubMed]

23. Li, C.-S.; An, C.-Y.; Li, X.-M.; Gao, S.-S.; Cui, C.-M.; Sun, H.-F.; Wang, B.-G. Triazole and dihydroimidazole alkaloids from the marine sediment-derived fungus Penicillium paneum SD-44. J. Nat. Prod. 2011, 74, 1331-1334. [CrossRef] [PubMed]

24. Xu, X.; Zhang, X.; Nong, X.; Wei, X.; Qi, S. Oxindole alkaloids from the fungus Penicillium commune DFFSCS026 isolated from deep-sea-derived sediments. Tetrahedron 2015, 71, 610-615. [CrossRef]

25. Wijesekara, I.; Li, Y.-X.; Vo, T.-S.; Van Ta, Q.; Ngo, D.-H.; Kim, S.-K. Induction of apoptosis in human cervical carcinoma HeLa cells by neoechinulin A from marine-derived fungus Microsporum sp. Process Biochem. 2013, 48, 68-72. [CrossRef]

26. Zhang, P.; Mandi, A.; Li, X.-M.; Du, F.-Y.; Wang, J.-N.; Li, X.; Kurtan, T.; Wang, B.-G. Varioxepine A, a $3 \mathrm{H}$-oxepine-containing alkaloid with a new oxa-cage from the marine algal-derived endophytic fungus Paecilomyces variotii. Org. Lett. 2014, 16, 4834-4837. [CrossRef]

27. Dasanayaka, S.; Nong, X.-H.; Liang, X.; Liang, J.-Q.; Amin, M.; Qi, S.-H. New dibenzodioxocinone and pyran-3, 5-dione derivatives from the deep-sea-derived fungus Penicillium canescens SCSIO z053. J. Asian Nat. Prod. Res. 2019, 1-8. [CrossRef]

28. Ren, J.; Xue, C.; Tian, L.; Xu, M.; Chen, J.; Deng, Z.; Proksch, P.; Lin, W. Asperelines A- F, peptaibols from the marine-derived fungus Trichoderma asperellum. J. Nat. Prod. 2009, 72, 1036-1044. [CrossRef]

29. Jiang, W.; Ye, P.; Chen, C.-T.; Wang, K.; Liu, P.; He, S.; Wu, X.; Gan, L.; Ye, Y.; Wu, B. Two novel hepatocellular carcinoma cycle inhibitory cyclodepsipeptides from a hydrothermal vent crab-associated fungus Aspergillus clavatus C2WU. Mar. Drugs 2013, 11, 4761-4772. [CrossRef]

30. Luo, X.W.; Yun, L.; Liu, Y.J.; Zhou, X.F.; Liu, Y.H. Peptides and polyketides isolated from the marine sponge-derived fungus Aspergillus terreus SCSIO 41008. Chin. J. Nat. Med. 2019, 17, 149-154. [CrossRef]

31. Liang, X.; Zhang, X.-Y.; Nong, X.-H.; Wang, J.; Huang, Z.-H.; Qi, S.-H. Eight linear peptides from the deep-sea-derived fungus Simplicillium obclavatum EIODSF 020. Tetrahedron 2016, 72, 3092-3097. [CrossRef]

32. Uchida, R.; Nakajyo, K.; Kobayashi, K.; Ohshiro, T.; Terahara, T.; Imada, C.; Tomoda, H. 7-Chlorofolipastatin, an inhibitor of sterol O-acyltransferase, produced by marine-derived Aspergillus ungui NKH-007. J. Antibiot. 2016, 69, 647. [CrossRef] [PubMed]

33. Pan, C.; Shi, Y.; Auckloo, B.N.; ul Hassan, S.S.; Akhter, N.; Wang, K.; Ye, Y.; Chen, C.-T.A.; Tao, X.; Wu, B. Isolation and antibiotic screening of fungi from a hydrothermal vent site and characterization of secondary metabolites from a Penicillium isolate. Mar. Biotechnol. 2017, 19, 469-479. [CrossRef] [PubMed] 
34. Huang, Z.; Nong, X.; Ren, Z.; Wang, J.; Zhang, X.; Qi, S. Anti-HSV-1, antioxidant and antifouling phenolic compounds from the deep-sea-derived fungus Aspergillus versicolor SCSIO 41502. Bioorg. Med. Chem. Lett. 2017, 27, 787-791. [CrossRef] [PubMed]

35. Liu, C.-C.; Zhang, Z.-Z.; Feng, Y.-Y.; Gu, Q.-Q.; Li, D.-H.; Zhu, T.-J. Secondary metabolites from Antarctic marine-derived fungus Penicillium crustosum HDN153086. Nat. Prod. Res. 2019, 33, 414-419. [CrossRef] [PubMed]

36. Orfali, R.S.; Aly, A.H.; Ebrahim, W.; Abdel-Aziz, M.S.; Müller, W.E.; Lin, W.; Daletos, G.; Proksch, P. Pretrichodermamide $\mathrm{C}$ and N-methylpretrichodermamide $\mathrm{B}$, two new cytotoxic epidithiodiketopiperazines from hyper saline lake derived Penicillium sp. Phytochem. Lett. 2015, 11, 168-172. [CrossRef]

37. Fan, Z.; Sun, Z.-H.; Liu, Z.; Chen, Y.-C.; Liu, H.-X.; Li, H.-H.; Zhang, W.-M. Dichotocejpins A-C: New diketopiperazines from a deep-sea-derived fungus Dichotomomyces cejpii FS110. Mar. Drugs 2016, 14, 164. [CrossRef]

38. Li, Y.; Ye, D.; Shao, Z.; Cui, C.; Che, Y. A sterol and spiroditerpenoids from a Penicillium sp. isolated from a deep sea sediment sample. Mar. Drugs 2012, 10, 497-508. [CrossRef]

39. Li, X.-D.; Li, X.-M.; Li, X.; Xu, G.-M.; Liu, Y.; Wang, B.-G. Aspewentins D-H, 20-nor-isopimarane derivatives from the deep sea sediment-derived fungus Aspergillus wentii SD-310. J. Nat. Prod. 2016, 79, 1347-1353. [CrossRef]

40. Wu, G.; Lin, A.; Gu, Q.; Zhu, T.; Li, D. Four new chloro-eremophilane sesquiterpenes from an Antarctic deep-sea derived fungus, Penicillium sp. PR19N-1. Mar. Drugs 2013, 11, 1399-1408. [CrossRef]

41. Lin, A.; Wu, G.; Gu, Q.; Zhu, T.; Li, D. New eremophilane-type sesquiterpenes from an antarctic deep-sea derived fungus, Penicillium sp. PR19 N-1. Arch. Pharm. Res. 2014, 37, 839-844. [CrossRef]

42. Zhou, Y.; Li, Y.-H.; Yu, H.-B.; Liu, X.-Y.; Lu, X.-L.; Jiao, B.-H. Furanone derivative and sesquiterpene from antarctic marine-derived fungus Penicillium sp. S-1-18. J. Asian Nat. Prod. Res. 2018, 20, 1108-1115. [CrossRef] [PubMed]

43. Li, X.-D.; Li, X.; Li, X.-M.; Xu, G.-M.; Zhang, P.; Meng, L.-H.; Wang, B.-G. Tetranorlabdane diterpenoids from the deep sea sediment-derived fungus Aspergillus wentii SD-310. Planta Med. 2016, 82, 877-881. [CrossRef] [PubMed]

44. Li, X.; Li, X.-M.; Li, X.-D.; Xu, G.-M.; Liu, Y.; Wang, B.-G. 20-Nor-isopimarane cycloethers from the deep-sea sediment-derived fungus Aspergillus wentii SD-310. RSC Adv. 2016, 6, 75981-75987. [CrossRef]

45. Fang, W.; Lin, X.; Zhou, X.; Wan, J.; Lu, X.; Yang, B.; Ai, W.; Lin, J.; Zhang, T.; Tu, Z. Cytotoxic and antiviral nitrobenzoyl sesquiterpenoids from the marine-derived fungus Aspergillus ochraceus Jcma1F17. MedChemComm 2014, 5, 701-705. [CrossRef]

46. Niu, S.; Fan, Z.-W.; Xie, C.-L.; Liu, Q.; Luo, Z.-H.; Liu, G.; Yang, X.-W. Spirograterpene A, a tetracyclic spiro-diterpene with a fused 5/5/5/5 ring system from the deep-sea-derived fungus Penicillium granulatum MCCC 3A00475. J. Nat. Prod. 2017, 80, 2174-2177. [CrossRef]

47. Chung, Y.-M.; Wei, C.-K.; Chuang, D.-W.; El-Shazly, M.; Hsieh, C.-T.; Asai, T.; Oshima, Y.; Hsieh, T.-J.; Hwang, T.-L.; Wu, Y.-C. An epigenetic modifier enhances the production of anti-diabetic and anti-inflammatory sesquiterpenoids from Aspergillus sydowii. Bioorg. Med. Chem. 2013, 21, 3866-3872. [CrossRef]

48. Li, C.-S.; Li, X.-M.; Gao, S.-S.; Lu, Y.-H.; Wang, B.-G. Cytotoxic anthranilic acid derivatives from deep sea sediment-derived fungus Penicillium paneum SD-44. Mar. Drugs 2013, 11, 3068-3076. [CrossRef]

49. Chen, L.; Gong, M.-W.; Peng, Z.-F.; Zhou, T.; Ying, M.-G.; Zheng, Q.-H.; Liu, Q.-Y.; Zhang, Q.-Q. The marine fungal metabolite, dicitrinone B, induces A375 cell apoptosis through the ROS-related caspase pathway. Mar. Drugs 2014, 12, 1939-1958. [CrossRef]

50. Pan, C.; Shi, Y.; Chen, X.; Chen, C.-T.A.; Tao, X.; Wu, B. New compounds from a hydrothermal vent crab-associated fungus Aspergillus versicolor XZ-4. Org. Biomol. Chem. 2017, 15, 1155-1163. [CrossRef]

51. Xu, R.; Xu, G.-M.; Li, X.-M.; Li, C.-S.; Wang, B.-G. Characterization of a newly isolated marine fungus Aspergillus dimorphicus for optimized production of the anti-tumor agent wentilactones. Mar. Drugs 2015, 13, 7040-7054. [CrossRef]

(C) 2019 by the authors. Licensee MDPI, Basel, Switzerland. This article is an open access article distributed under the terms and conditions of the Creative Commons Attribution (CC BY) license (http://creativecommons.org/licenses/by/4.0/). 